\title{
The Spectrum for 3-way $k$-homogeneous Latin Trades
}

\author{
Trent G. Marbach \\ Department of Mathematics \\ The University of Queensland \\ Brisbane 4072, Australia \\ trent.marbach@uqconnect.edu.au
}

\author{
Lijun $\mathrm{Ji}^{*}$ \\ Department of Mathematics \\ Soochow University \\ Suzhou 215006, China \\ jilijun@suda.edu.cn
}

Submitted: Mar 23, 2015; Accepted: Sep 17, 2015; Published: Oct 16, 2015

Mathematics Subject Classifications: 05B15

\begin{abstract}
A $\mu$-way $k$-homogeneous Latin trade was defined by Bagheri Gh, Donovan, Mahmoodian (2012), where the existence of 3 -way $k$-homogeneous Latin trades was specifically investigated. We investigate the existence of a certain class of $\mu$-way $k$-homogeneous Latin trades with an idempotent like property. We present a number of constructions for $\mu$-way $k$-homogeneous Latin trades with this property, and show that these can be used to fill in the spectrum of 3-way $k$-homogeneous Latin trades for all but 196 possible exceptions.
\end{abstract}

Keywords: Latin square; Latin trade; $\mu$-way $k$-homogeneous Latin trade

\section{Introduction}

A partial Latin square of order $m, T=[t(r, c)]$, is an $m \times m$ array of cells with each cell either filled with an element $t(r, c)$ of $\Omega$ (a set of $m$ symbols) or left empty, such that each symbol of $\Omega$ appears at most once in each row, and at most once in each column. In what follows, we typically take $\Omega=[m]=\{1,2, \ldots, m\}$ and index the rows and columns of the partial Latin square by $[m]$. A partial Latin square $T$ has volume $s$ if it has precisely $s$ filled cells, where $0 \leqslant s \leqslant m^{2}$. A partial Latin square with volume $m^{2}$ is a Latin square. We can represent $T$ as a set of $s$ ordered triples $\{(r, c, t(r, c)) \mid r, c \in[m]$ and cell $(r, c)$ is not empty $\}$. The back-circulant Latin squares are defined as $B_{m}=\{(i, j, i+j \bmod m) \mid i, j \in[m]\}$. A diagonal of a Latin square $L$ is a set of $m$ cells of $L$ such that each row and each column is represented in the set of cells

${ }^{*}$ Research supported by NSFC grant 11222113, 11431003 (L. Ji) 
precisely once. A transversal of a Latin square is a diagonal that also has each symbol represented precisely once in the diagonal.

The shape of $T$ is $\mathcal{S}(T)=\left\{\left(r^{\prime}, c^{\prime}\right) \in[m] \times[m] \mid\left(r^{\prime}, c^{\prime}, e\right) \in T\right.$, for some $\left.e \in[m]\right\}$. The $r$-th row set of $T$ is defined as $\mathcal{R}_{r}(T)=\left\{e^{\prime} \in[m] \mid\left(r, c^{\prime}, e^{\prime}\right) \in T\right.$, for some $\left.c^{\prime} \in[m]\right\}$. The c-th column set of $T$ is defined as $\mathcal{C}_{c}(T)=\left\{e^{\prime} \in[m] \mid\left(r^{\prime}, c, e^{\prime}\right) \in T\right.$, for some $\left.r^{\prime} \in[m]\right\}$. The $e$-th symbol set of $T$ is defined as $\mathcal{E}_{e}(T)=\left\{\left(r^{\prime}, c^{\prime}\right) \in[m] \times[m] \mid\left(r^{\prime}, c^{\prime}, e\right) \in T\right\}$.

Definition 1. For natural numbers $\mu, m, \mu \leqslant m$, a $\mu$-way Latin trade of order $m$ on symbol set $\Omega$ is a collection $\mathcal{T}=\left(T_{1}, \ldots, T_{\mu}\right)$ of $\mu$ partial Latin squares of order $m$ using symbols of $\Omega$ such that:

- $\mathcal{S}\left(T_{\alpha}\right)=\mathcal{S}\left(T_{\beta}\right)$, for each $1 \leqslant \alpha<\beta \leqslant \mu ;$

- for each $(r, c) \in \mathcal{S}\left(T_{\alpha}\right)$ it holds that $t_{\alpha}(r, c) \neq t_{\beta}(r, c)$, for every $1 \leqslant \alpha<\beta \leqslant \mu$; and

- $\mathcal{R}_{r}\left(T_{\alpha}\right)=\mathcal{R}_{r}\left(T_{\beta}\right)$ and $\mathcal{C}_{c}\left(T_{\alpha}\right)=\mathcal{C}_{c}\left(T_{\beta}\right)$, for each $r, c \in[m]$ and $1 \leqslant \alpha<\beta \leqslant \mu$.

Let $\mathcal{T}=\left(T_{1}, \ldots, T_{\mu}\right)$ be a $\mu$-way Latin trade. As the shape of each $T_{\alpha}$ is the same, we can define the shape of $\mathcal{T}$ as $\mathcal{S}(\mathcal{T})=\mathcal{S}\left(T_{1}\right)$. Then the volume of $\mathcal{T}$ is the volume of $T_{1}$. Similarly, the row sets (resp. column sets and symbol sets) of each $T_{\alpha}$ are the same, so we can define a row set for row $r$ (resp. column set for column $c$ and symbol set for symbol e) of $\mathcal{T}$ as $\mathcal{R}_{r}(\mathcal{T})=\mathcal{R}_{r}\left(T_{1}\right)\left(\operatorname{resp} . \mathcal{C}_{c}(\mathcal{T})=\mathcal{C}_{c}\left(T_{1}\right)\right.$ and $\mathcal{E}_{e}(\mathcal{T})=\mathcal{E}_{e}\left(T_{1}\right)$ ).

Definition 2. For an integer $k \geqslant 0$, a $(\mu, k, m)$-Latin trade on symbol set $\Omega, \mathcal{T}=$ $\left(T_{1}, \ldots, T_{\mu}\right)$, is a $\mu$-way Latin trade of order $m$ on $\Omega$ that has $k=\left|\mathcal{R}_{r}(\mathcal{T})\right|=\left|\mathcal{C}_{c}(\mathcal{T})\right|=$ $\left|\mathcal{E}_{e}(\mathcal{T})\right|$, for each $r, c, e \in[m]$. Such a $\mu$-way Latin trade is called $k$-homogeneous.

A $(\mu, k, m)$-Latin trade can have $k=0$ in the case that each of the $\mu$ partial Latin squares is empty; otherwise $k$ must satisfy $\mu \leqslant k \leqslant m$.

We will require the $(\mu, k, m)$-Latin trades that we investigate to have the property that if $(r, c, e) \in T_{\alpha}$, where $T_{\alpha}$ is one of the partial Latin squares that form a $(\mu, k, m)$-Latin trade, then $r, c, e$ are pairwise distinct. With this property, $T_{\alpha} \cup\{(i, i, i) \mid i \in[m]\}$ would form a new partial Latin square that resembles an idempotent Latin square with some unfilled cells.

Definition 3. A $\mu$-way Latin trade $\mathcal{T}$ of order $m$ is idempotent if $i \notin \mathcal{R}_{i}(\mathcal{T}) \cup \mathcal{C}_{i}(\mathcal{T})$ and $(i, i) \notin \mathcal{S}(\mathcal{T})$, for $i \in[m]$.

Definition 4. A $(\mu, k, m)$-Latin trade is circulant if it can be obtained from the elements of its first row, called the base row (denoted by $\mu-B_{m}^{k}$ ), by simultaneously permuting each of the coordinates cyclically. That is, for each $\alpha$, the cell $(1, c, e) \in T_{\alpha}$ implies $(1+i, c+i$ $\bmod m, e+i \bmod m) \in T_{\alpha}$, for $1 \leqslant i \leqslant m-1$.

We write the base row as $B=\left\{\left(a_{1}, \ldots, a_{\mu}\right)_{c_{l}} \mid 1 \leqslant l \leqslant k\right\}$, where $a_{\alpha}, c_{l} \in[m]$. Then the corresponding $\mu$ partial Latin squares can be constructed as $T_{\alpha}=\left\{\left(1+i, c_{l}+i\right.\right.$ $\left.\left.\bmod m, a_{\alpha}+i \bmod m\right) \mid 0 \leqslant i \leqslant m-1,1 \leqslant l \leqslant k\right\}, \alpha \in[\mu]$. We will denote an idempotent circulant $(\mu, k, m)$-Latin trade by $\mu-I B_{m}^{k}$. 
Let $m$ be an integer. The spectrum of $\mu$-way homogeneous Latin trades of order $m$, $\mathcal{S}_{m}^{\mu}$, is the set of values of $k$ such that there exists a $(\mu, k, m)$-Latin trade. The spectrum of idempotent $\mu$-way homogeneous Latin trades of order $m, \mathcal{I} \mathcal{S}_{m}^{\mu}$, is the set of values of $k$ such that there exists an idempotent $(\mu, k, m)$-Latin trade.

A previous study of $(\mu, k, m)$-Latin trades [2] posed the question:

Question 5. For given $m$ and $k, m \geqslant k \geqslant \mu$, does there exist a $(\mu, k, m)$-Latin trade?

The primary goal of this paper is to investigate this question by deducing $\mathcal{S}_{m}^{3}$. However in order to do this, we will use a construction that requires us to first investigate $\mathcal{I S}_{m}^{3}$. Clearly $\mathcal{I S}_{m}^{3} \subseteq \mathcal{S}_{m}^{3}$. It is known that $\{3, \ldots, m\} \supseteq \mathcal{S}_{m}^{3}$, and also that $3 \in \mathcal{S}_{m}^{3}$ if and only if $3 \mid m$ (see [2]). In this paper, we show there exists 3 -way $k$-homogeneous Latin trades of order $m$ with $4 \leqslant k \leqslant m$ for all but a finite list of possible exceptions.

\section{Literature review}

A 2-way Latin trade is typically called a Latin bitrade. There have been three distinct approaches used to construct $k$-homogeneous Latin bitrades.

The first approach used graph theoretic constructions (see also [5], [12], [13], and [14]):

Theorem 6. $[6,7]$ There exists a $(2, p, 3 m)$-Latin trade when $p=3,4$ and $m \geqslant 3$.

The second approach used block theoretic based constructions:

Theorem 7. [3, 4] There exists $a(2, k, m)$-Latin trade when $3 \leqslant k \leqslant 37$ and $m \geqslant k$.

The third approach relies on finding pairs of transversals of given intersection in the back-circulant Latin squares:

Theorem 8. [8] For each odd $m \neq 5$ and for each $t \in\{0, \ldots, m-3\} \cup\{m\}$, there exists two transversals in $B_{m}, T_{1}$ and $T_{2}$, with $\left|T_{1} \cap T_{2}\right|=t$. When $m=5$ and for each $t \in\{0,1,5\}$, there exists two transversals in $B_{m}, T_{1}$ and $T_{2}$, with $\left|T_{1} \cap T_{2}\right|=t$.

Lemma 9. [8] For $m$ an odd integer, let $T_{1}$ and $T_{2}$ be two transversals in $B_{m}$ such that $\left|T_{1} \cap T_{2}\right|=t$. Then there exists a $(2, m-t, m)$-Latin trade.

These results lead to the completion of the spectrum problem for homogeneous Latin bitrades:

Theorem 10. $[3,4,6,7,8]$ There is a $(2, k, m)$-Latin trade for all $3 \leqslant k \leqslant m$ and $a$ $(2,2,2 m)$-Latin trade, for all $m \geqslant 1$.

The first study of $(\mu, k, m)$-Latin trades for general $\mu$ produced a number of block theoretic constructions [2] that yielded results for small $k$ when $\mu=3$ :

Theorem 11. [2] There exist $(3, k, m)$-Latin trades for $m \geqslant k$ when:

- $k=3$ and $3 \mid m$; 
- $k=4$ and $m \neq 6,7,11$;

- $5 \leqslant k \leqslant 13$;

- $k=15$; and

- $k=m$.

\section{Idempotent generalization of basic constructions}

The constructions that have appeared earlier in the literature for $(\mu, k, m)$-Latin trades [2] can be used (or modified) for the construction of idempotent $(\mu, k, m)$-Latin trades. As many of the constructions differ only trivially from their original appearance, we label the source of the original construction, and give the original proof with an extension if necessary.

Theorem 12. [2] If there exist idempotent $\left(\mu, k, m_{i}\right)$-Latin trades, for $i=1,2$, then there exists an idempotent $\left(\mu, k, m_{1}+m_{2}\right)$-Latin trade.

Theorem 13. [2] If there exist an idempotent $\left(\mu_{1}, k_{1}, m_{1}\right)$-Latin trade and a $\left(\mu_{2}, k_{2}, m_{2}\right)$ Latin trade, then there exists an idempotent $\left(\mu_{1} \mu_{2}, k_{1} k_{2}, m_{1} m_{2}\right)$-Latin trade.

Theorem 14. [2] If $l \neq 2,6$ and for each $k \in\left\{k_{2}, \ldots, k_{l}\right\}$ there exists a $(\mu, k, p)$-Latin trade and there exists an idempotent $\left(\mu, k_{1}, p\right)$-Latin trade, then an idempotent $\left(\mu, k_{1}+\right.$ $\left.\cdots+k_{l}, l p\right)$-Latin trade exists. (Some $k_{i}$ 's can possibly be zero.)

Proof. For $l \neq 2,6$, there exists two $l \times l$ orthogonal Latin squares. Denote these Latin squares by $L_{1}$ and $L_{2}$, with elements chosen from the sets $\left\{e_{1}, \ldots, e_{l}\right\}$ and $\left\{f_{1}, \ldots, f_{l}\right\}$, respectively. We can simultaneously permute the rows and columns of $L_{1}$ and $L_{2}$ so the main diagonal of $L_{2}$ contains only $f_{1}$, and then re-label the symbols of $L_{1}$ so that the symbols in cell $(j, j)$ of $L_{1}$ is $e_{j}$. Assume that $L^{*}$ is the square that is formed by superimposing $L_{1}$ and $L_{2}$. We replace each $\left(e_{i}, f_{j}\right) \in L^{*}$ such that $j \geqslant 2$ with a $\left(\mu, k_{j}, p\right)$ Latin trade whose elements are from the set $\{(i-1) p+1, \ldots, i p\}$, and when $j=1$ with an idempotent $\left(\mu, k_{1}, p\right)$-Latin trade whose elements are from the set $\{(i-1) p+1, \ldots, i p\}$. As a result we obtain a $\left(\mu, k_{1}+\cdots+k_{l}, l p\right)$-Latin trade, which we denote as $\mathcal{T}$.

Then clearly $(j, j) \notin \mathcal{S}(\mathcal{T})$ as each of the entries on the main diagonal of $\mathcal{T}$ came from an idempotent $\left(\mu, k_{1}, p\right)$-Latin trade. Note that in $\mathcal{T}$ a row $r \in\{(i-1) p+1, \ldots, i p\}$ contains cells filled with symbols $e \in\{(i-1) p+1, \ldots, i p\}$ only in columns $c \in\{(i-$ $1) p+1, \ldots, i p\}$, and these filled cells came from an idempotent $(\mu, k, p)$-Latin trade. So if $(i-1) p+i^{\prime} \in \mathcal{R}_{(i-1) p+i^{\prime}}(\mathcal{T}), 1 \leqslant i^{\prime} \leqslant p$, then there must be a cell in row $(i-1) p+i^{\prime}$ and column $c$ with $c \in\{(i-1) p+1, \ldots, i p\}$ that contains symbol $(i-1) p+i^{\prime}$, and this comes from an idempotent $(\mu, k, m)$-Latin trade, say $\mathcal{U}$. But then $\mathcal{U}$ would have $i^{\prime} \in \mathcal{R}_{i^{\prime}}(\mathcal{U})$, a contradiction as $\mathcal{U}$ is idempotent. The analogous result holds for the columns, and $\mathcal{T}$ forms an idempotent $\left(\mu, k_{1}+\cdots+k_{l}, l p\right)$-Latin trade. 
Theorem 15. Take $k$ and $k^{\prime}$ to be integers with $k^{\prime}>k$. If for every $k^{\prime} \leqslant l \leqslant 2 k^{\prime}-1$ there exists an idempotent $(\mu, k, l)$-Latin trade, then for any $m \geqslant k^{\prime}$ there exists an idempotent $(\mu, k, m)$-Latin trade.

Proof. For every $m \geqslant 2 k^{\prime}$, we can write $m=r k^{\prime}+s l$, for some $r, s \geqslant 0$ and $k^{\prime}+1 \leqslant l \leqslant$ $2 k^{\prime}-1$. Since there exist an idempotent $\left(\mu, k, k^{\prime}\right)$-Latin trade and an idempotent $(\mu, k, l)$ Latin trade, by Theorem 12 we conclude that there exists an idempotent $(\mu, k, m)$-Latin trade.

A large set of idempotent Latin squares of order $m$ is a set of $m-2$ idempotent Latin squares of order $m,\left(L_{1}, \ldots, L_{m-2}\right)$, such that for $\alpha, \beta$ with $1 \leqslant \alpha<\beta \leqslant m-2$ and $i, j \in[m], L_{\alpha}(i, i)=L_{\beta}(i, i)=i$ and $L_{\alpha}(i, j) \neq L_{\beta}(i, j)$ when $i \neq j$.

Theorem 16. For $m \geqslant 3, m \neq 6$, there exists an idempotent $(\mu, m-1, m)$-Latin trade whenever $1 \leqslant \mu \leqslant m-2$.

Proof. It was shown in [17] that for $m \neq 6,14,62$ there exists a large set of idempotent Latin squares of order $m$. The cases $m=14,62$ were solved in [10] and [9] respectively. By taking such a large set and deleting the cells of the main diagonals of each of the idempotent Latin squares, we have an idempotent $(m-2, m-1, m)$-Latin trade for $m \geqslant 3, m \neq 6$. Clearly we can remove any number of the resulting partial Latin squares to yield an idempotent $(\mu, m-1, m)$-Latin trade for $1 \leqslant \mu \leqslant m-2$.

Generalizing from the method of finding pairs of transversals of given intersection in the back-circulant Latin squares, the author of [16] was able to determine the possible intersection sizes of three transversals in the back circulant Latin square:

Theorem 17. [16] For odd integer $m \geqslant 33$ with $m=18 I+9+2 d, 0 \leqslant d<9$, and $m=22 I^{\prime}+11+2 d^{\prime}, 0 \leqslant d^{\prime}<11$, there exists three transversals of the back circulant Latin square $B_{m}, T_{1}, T_{2}, T_{3}$, for each $t \in\left\{\min \left(d^{\prime}+3, d\right), \ldots, m\right\} \backslash\{m-5, \ldots, m-1\}$ such that $S=T_{1} \cap T_{2}=T_{1} \cap T_{3}=T_{2} \cap T_{3}$ and $|S|=t$, except possibly when:

- $m=51$ and $t=29$,

- $m=53$ and $t=30$.

Further, a transformation is provided to construct $(\mu, k, m)$-Latin trades from a collection of $\mu$ transversals of the back circulant Latin square.

Theorem 18. [16] Take $m$ odd and $0 \leqslant t \leqslant m$. If there exists a set $S \subseteq[m]^{3}$ with $|S|=t$ and $\mu$ transversals of $B_{m}, T_{1}, \ldots, T_{\mu}$ with $T_{\alpha} \cap T_{\beta}=S$, for $\alpha, \beta \in[\mu]$ and $\alpha \neq \beta$, then there exists a circulant $(\mu, m-t, m)$-Latin trade.

Proof. Consider the $\mu$ partial Latin squares defined by $Q_{\alpha}=\{(i, c+i, r+c+i) \mid i \in$ $\left.[m],(r, c, r+c) \in T_{\alpha} \backslash S\right\}$. The set $\left(Q_{1}, \ldots, Q_{\mu}\right)$ forms a $(\mu, m-t, m)$-Latin trade that is circulant by definition.

This can be generalized for our purposes in the following manner: 
Theorem 19. Take $m$ odd and $1 \leqslant t \leqslant m$. If there is a set $S \subseteq[m]^{3}$ with $|S|=t$ such that there exists $\mu$ transversals of $B_{m}, T_{1}, \ldots, T_{\mu}$, with $T_{\alpha} \cap T_{\beta}=S$, for $\alpha, \beta \in[m]$ and $\alpha \neq \beta$, then there exists an idempotent circulant $(\mu, m-t, m)$-Latin trade.

Proof. Notice that $T_{\alpha}^{x, y}=\left\{(r+x, c+y, r+c+x+y) \mid(r, c, r+c) \in T_{\alpha}\right\}$, for $\alpha \in[\mu]$ and $x, y \in\{0, \ldots, m-1\}$, will define a new collection of $\mu$ transversals of $B_{m}$ with $T_{\alpha}^{x, y} \cap T_{\beta}^{x, y}=S^{x, y}$ such that $\left|S^{x, y}\right|=t$, for $\alpha, \beta \in[\mu]$ and $\alpha \neq \beta$. As $t \geqslant 1$, this allows us to assume without loss of generality that $(m, m, m) \in T_{\alpha}$, for all $\alpha \in[\mu]$, or equivalently $(m, m, m) \in S$. Applying the construction from Theorem 18 to these transversals of $B_{m}$, we obtain a circulant $(\mu, m-t, m)$-Latin trade, which we denote as $\mathcal{Q}=\left(Q_{1}, \ldots, Q_{\mu}\right)$. As $(m, m, m) \in S$, then each $(r, c, r+c) \in T_{\alpha} \backslash S$ has $r+1 \not \equiv 1, c+1 \not \equiv 1$, and $r+c+1 \not \equiv 1$. Then $\mathcal{Q}$ has its $(1,1)$ cell empty as $c+1 \not \equiv 1$, and the symbol 1 will not appear in the first row as $r+c+1 \not \equiv 1$. The first column of $Q_{\alpha}$ contains cells $\left\{(m-c+1,1, r+1) \mid(r, c, r+c) \in T_{\alpha} \backslash S\right\}$, for $\alpha \in[\mu]$. As $r+1 \not \equiv 1$, the symbol 1 does not appear in the first column. By the circulant nature, we also have $(i, i) \notin \mathcal{S}(\mathcal{Q})$ and $i \notin \mathcal{R}_{i}(\mathcal{Q}) \cap \mathcal{C}_{i}(\mathcal{Q})$, for $i \in[m]$. Then $\mathcal{Q}$ is an idempotent circulant $(\mu, m-t, m)$-Latin trade.

We can then exploit the existence of two [8] and three [16] transversals of given intersection as:

Theorem 20. For odd integer $m \geqslant 5$, there exists an idempotent circulant $(2, m-t, m)$ Latin trade for $t \in[m] \backslash\{m-2, m-1\}$ except, perhaps, when $(t, m)=(2,5)$.

Theorem 21. For odd integer $m \geqslant 33$ with $m=18 I+9+2 d, 0 \leqslant d<9$, and $m=$ $22 I^{\prime}+11+2 d^{\prime}, 0 \leqslant d^{\prime}<11$, there exists an idempotent circulant $(3, m-t, m)$-Latin trade, for $t \in\left\{\max \left(1, \min \left(d^{\prime}+3, d\right)\right), \ldots, m\right\} \backslash\{m-5, \ldots, m-1\}$, except, perhaps, when:

- $m=51$ and $t=29$,

- $m=53$ and $t=30$.

\section{New constructions for idempotent $(\mu, k, m)$-Latin trades}

In this section, we will consider block theoretic constructions that are able to determine the spectrum of $(3, k, m)$-Latin trades for all but a small list of values of $k$ and $m$.

\subsection{Computer search for small orders}

If $B=\left\{\left(a_{1}, \ldots, a_{\mu}\right)_{c_{l}} \mid 1 \leqslant l \leqslant k\right\}$, Algorithm 1 of [2] can be used to show $B$ is the base row of a $(\mu, k, m)$-Latin trade. If for each $\left(a_{1}, \ldots, a_{\mu}\right)_{c_{l}}$ it further holds that:

- $a_{\alpha} \neq 1$, for all $\alpha \in[\mu]$;

- $c_{l} \neq 1$; and

- $a_{\alpha} \neq c_{l}$, 
then $B$ is the base row of an idempotent $(\mu, k, m)$-Latin trade. As the result of a computational search, we introduce the following base rows of idempotent $(3, k, m)$-Latin trades:

- $k=5$

$$
\begin{aligned}
3-I B_{7}^{5} & =\left\{(3,4,5)_{2},(5,7,4)_{3},(7,5,2)_{4},(2,3,7)_{5},(4,2,3)_{6}\right\} \\
3-I B_{8}^{5} & =\left\{(3,4,6)_{2},(8,2,4)_{3},(6,8,3)_{4},(4,6,2)_{5},(2,3,8)_{6}\right\} \\
3-I B_{9}^{5} & =\left\{(3,6,9)_{2},(6,2,7)_{3},(2,7,3)_{4},(9,3,6)_{5},(7,9,2)_{8}\right\} \\
3-I B_{11}^{5} & =\left\{(5,7,9)_{2},(7,2,8)_{3},(9,8,7)_{4},(2,9,5)_{6},(8,5,2)_{9}\right\} \\
3-I B_{12}^{5} & =\left\{(4,5,8)_{2},(11,2,5)_{3},(8,11,4)_{5},(5,8,2)_{6},(2,4,11)_{8}\right\}
\end{aligned}
$$

- $k=6$

$$
\begin{aligned}
3-I B_{8}^{6} & =\left\{(3,4,6)_{2},(5,8,2)_{3},(8,2,5)_{4},(2,6,3)_{5},(4,5,8)_{6},(6,3,4)_{7}\right\} \\
3-I B_{9}^{6} & =\left\{(3,6,7)_{2},(7,4,5)_{3},(6,2,3)_{4},(4,7,6)_{5},(2,5,4)_{6},(5,3,2)_{7}\right\} \\
3-I B_{10}^{6} & =\left\{(5,8,9)_{2},(9,2,4)_{3},(2,5,3)_{4},(4,3,8)_{5},(3,9,2)_{6},(8,4,5)_{7}\right\} \\
3-I B_{11}^{6} & =\left\{(3,4,10)_{2},(6,9,4)_{3},(10,2,6)_{4},(2,6,3)_{5},(4,3,9)_{6},(9,10,2)_{7}\right\} \\
3-I B_{12}^{6} & =\left\{(3,9,11)_{2},(7,2,5)_{3},(11,5,3)_{4},(2,7,9)_{5},(5,3,7)_{6},(9,11,2)_{7}\right\} \\
3-I B_{13}^{6} & =\left\{(8,11,13)_{2},(13,8,9)_{3},(9,2,8)_{4},(3,9,2)_{5},(2,3,11)_{6},(11,13,3)_{7}\right\}
\end{aligned}
$$

- $k=7$

$$
\begin{aligned}
3-I B_{m}^{7}= & \left\{(3,4,6)_{2},(7,6,5)_{3},(6,2,7)_{4},(2,9,3)_{5},(9,7,2)_{6},(5,3,4)_{7},(4,5,9)_{8}\right\}, \\
& \text { for } m \geqslant 9
\end{aligned}
$$

- $k=8$

$$
\begin{aligned}
3-I B_{m}^{8}= & \left\{(3,4,5)_{2},(2,6,7)_{3},(7,8,2)_{4},(9,2,6)_{5},(8,5,3)_{6},(4,3,9)_{7},(6,9,4)_{8},\right. \\
& \left.(5,7,8)_{9}\right\}, \text { for } m \geqslant 10
\end{aligned}
$$

- $k=9$

$$
\begin{aligned}
3-I B_{m}^{9}= & \left\{(3,4,5)_{2},(5,8,7)_{3},(7,2,9)_{4},(9,6,2)_{5},(11,9,8)_{6},(2,11,3)_{7},(4,3,6)_{8},\right. \\
& \left.(6,5,4)_{9},(8,7,11)_{10}\right\}, \text { for } m \geqslant 11
\end{aligned}
$$

- $k=10$

$$
\begin{aligned}
3-I B_{m}^{10}= & \left\{(3,4,5)_{2},(2,6,4)_{3},(6,5,9)_{4},(9,10,2)_{5},(11,2,10)_{6},(10,11,3)_{7},(4,3,7)_{8}\right. \\
& \left.(7,8,11)_{9},(5,7,8)_{10},(8,9,6)_{11}\right\}, \text { for } m \geqslant 12
\end{aligned}
$$

- $k=11$

$$
\begin{aligned}
3-I B_{m}^{11}= & \left\{(3,4,5)_{2},(5,6,8)_{3},(7,2,10)_{4},(9,11,3)_{5},(11,10,2)_{6},(13,8,11)_{7},\right. \\
& \left.(2,13,9)_{8},(4,3,6)_{9},(6,5,4)_{10},(8,7,13)_{11},(10,9,7)_{12}\right\}, \text { for } m \geqslant 13
\end{aligned}
$$


- $k=12$

$$
\begin{aligned}
3-I B_{m}^{12}= & \left\{(3,4,5)_{2},(2,6,4)_{3},(6,2,3)_{4},(8,9,11)_{5},(11,12,8)_{6},(13,3,12)_{7},\right. \\
& \left.(12,13,2)_{8},(4,10,13)_{9},(7,5,6)_{10},(5,8,9)_{11},(10,11,7)_{12},(9,7,10)_{13}\right\}, \\
& \text { for } m \geqslant 14
\end{aligned}
$$

- $k=13$

$$
\begin{aligned}
3-I B_{m}^{13}= & \left\{(3,4,5)_{2},(5,6,4)_{3},(2,5,10)_{4},(9,10,12)_{5},(11,13,2)_{6},(13,2,9)_{7},\right. \\
& (15,12,13)_{8},(12,15,3)_{9},(4,3,8)_{10},(6,7,15)_{11},(8,9,7)_{12},(10,11,6)_{13}, \\
& \left.(7,8,11)_{14}\right\}, \text { for } m \geqslant 15
\end{aligned}
$$

- $k=14$

$$
\begin{aligned}
3-I B_{m}^{14}= & \left\{(3,4,5)_{2},(2,6,4)_{3},(6,2,3)_{4},(8,9,2)_{5},(10,3,13)_{6},(13,14,12)_{7},\right. \\
& (15,13,14)_{8},(14,15,11)_{9},(4,5,6)_{10},(7,12,15)_{11},(5,11,10)_{12}, \\
& \left.(11,7,8)_{13},(9,10,7)_{14},(12,8,9)_{15}\right\}, \text { for } m \geqslant 16
\end{aligned}
$$

- $k=15$

$$
\begin{aligned}
3-I B_{m}^{15}= & \left\{(3,4,5)_{2},(5,6,4)_{3},(2,5,6)_{4},(8,2,9)_{5},(11,13,14)_{6},(13,15,12)_{7},\right. \\
& (15,12,2)_{8},(17,14,15)_{9},(14,3,17)_{10},(4,17,3)_{11},(6,7,10)_{12}, \\
& \left.(10,11,8)_{13},(9,10,7)_{14},(7,9,11)_{15},(12,8,13)_{16}\right\}, \text { for } m \geqslant 17
\end{aligned}
$$

- $k=16$

$$
\begin{aligned}
3-I B_{m}^{16}= & \left\{(3,4,5)_{2},(2,6,4)_{3},(6,2,3)_{4},(8,9,2)_{5},(4,3,10)_{6},(13,12,15)_{7},\right. \\
& (15,16,13)_{8},(17,15,16)_{9},(14,17,6)_{10},(16,5,17)_{11},(5,7,14)_{12}, \\
& \left.(7,14,8)_{13},(10,13,12)_{14},(12,11,7)_{15},(11,8,9)_{16},(9,10,11)_{17}\right\} \\
& \text { for } m \geqslant 18
\end{aligned}
$$

- $k=17$

$$
\begin{aligned}
3-I B_{19}^{17}= & \left\{(3,4,5)_{2},(5,6,4)_{3},(2,5,6)_{4},(8,2,3)_{5},(10,11,12)_{6},(13,14,15)_{7},\right. \\
& (15,17,19)_{8},(17,13,14)_{9},(19,16,17)_{10},(16,19,2)_{11},(4,3,16)_{12}, \\
& (6,8,7)_{13},(9,7,11)_{14},(11,9,10)_{15},(7,12,9)_{16},(14,15,13)_{17}, \\
& \left.(12,10,8)_{18}\right\} \\
3-I B_{m}^{17}= & (3,4,5)_{2},(2,6,4)_{3},(6,2,3)_{4},(8,9,7)_{5},(10,5,11)_{6},(14,15,16)_{7}, \\
& (16,17,15)_{8},(18,14,17)_{9},(15,16,14)_{10},(17,18,2)_{11},(4,13,18)_{12}, \\
& (7,3,8)_{13},(12,8,10)_{14},(5,11,13)_{15},(11,7,6)_{16},(13,12,9)_{17}, \\
& \left.(9,10,12)_{18}\right\}, \text { for } m \geqslant 20
\end{aligned}
$$


- $k=18$

$$
\begin{aligned}
3-I B_{m}^{18}= & \left\{(3,4,5)_{2},(2,6,4)_{3},(6,2,3)_{4},(8,9,2)_{5},(4,3,8)_{6},(11,8,12)_{7},\right. \\
& (15,16,17)_{8},(17,18,16)_{9},(19,15,18)_{10},(16,17,15)_{11},(18,19,6)_{12}, \\
& (5,7,19)_{13},(7,5,9)_{14},(9,14,13)_{15},(12,11,7)_{16},(14,13,10)_{17}, \\
& \left.(13,10,14)_{18},(10,12,11)_{19}\right\}, \text { for } m \geqslant 20
\end{aligned}
$$

Theorem 22. There exist idempotent $(3, k, m)$-Latin trades for $m \geqslant k+1$ when:

- $k=4$ and $5 \mid m ;$

- $k=5$, except when $m=6$ and perhaps when $m=10,13$; and

- $6 \leqslant k \leqslant 18$.

Proof. The previously stated base rows, along with the idempotent $(3, m-1, m)$-Latin trades of Theorem 16 complete the cases for $7 \leqslant k \leqslant 18$. For $k=6$, we can use the previously stated base rows along with an idempotent $(3,6,7)$-Latin trade from Theorem 16 with Theorem 15. For $k=5$, there exists idempotent $(3,5, m)$-Latin trades for $m \in$ $\{7,8,9,11,12\}$. Using these $(3,5, m)$-Latin trades in Theorem 12 will, after two iterations, yield the required $(3,5, m)$-Latin trades for Theorem 15 with $k^{\prime}=14$. For $k=4$, there exists an idempotent $(3,4,5)$-Latin trade by Theorem 16, on which we can repeatedly apply Theorem 12 to get the result. If an idempotent $(3,5,6)$-Latin trade existed, then adding the cells $\{(i, i, i) \mid 1 \leqslant i \leqslant n\}$ to each of the partial Latin squares of the idempotent $(3,5,6)$-Latin trade would yield an ordered triple of idempotent Latin squares of order 6 that are pairwise disjoint in each cell not on the main diagonal. Using a computer we searched for such ordered triples of idempotent Latin squares by testing all possible triples of idempotent Latin squares of order 6 . None existed, and so there does not exists an idempotent $(3,5,6)$-Latin trade.

We conjecture that the two unresolved cases with $k=5$ and $m=10,13$ both exist.

\subsection{Extended multiplication construction}

Lemma 23. Take $n \geqslant 3$ and $m \geqslant 4$. If $n=6$, let $y$ be a positive integer with $\mu<y \leqslant \frac{m}{4}$. If $n \neq 6$, let $y$ be a positive integer with $\mu<y \leqslant \frac{m}{2}$. If there exists an idempotent $(\mu, k, m)$-Latin trade for each $k \in\{0, y, y+1, \ldots, m-1\}$, then there exists an idempotent $(\mu, k, m n)$-Latin trade for each $k \in\{0, y, y+1, \ldots, m n-1\}$.

Proof. In the case that $n \neq 6$, Theorem 14 yields idempotent $(\mu, k, m n)$-Latin trades for $k \in\left\{\sum_{i=1}^{n} y_{i} \mid y_{1} \in\{0, y, y+1, \ldots, m-1\}\right.$ and $\left.y_{i} \in\{0, y, y+1, \ldots, m\}, 2 \leqslant i \leqslant n\right\}=$ $\{0, y, y+1, \ldots, m n-1\}\left(y_{i}\right.$, with $2 \leqslant i \leqslant n$, may equal $m$ as there exists a $(3, m, m)$-Latin trade by Theorem 11).

Now we consider the case when $n=6$. Applying Theorem 13 using an idempotent $(\mu, k, m)$-Latin trade and a $(1,2,2)$-Latin trade (which exists by Theorem 10) yields an 
idempotent $(\mu, 2 k, 2 m)$-Latin trade for each $k$ with $y \leqslant k \leqslant m-1$. Applying Theorem 12 to the idempotent $(\mu, k, m)$-Latin trades yields idempotent $(\mu, k, 2 m)$-Latin trades for each $k$ with $y \leqslant k \leqslant m-1$. Considering these, along with the existence of an idempotent $(\mu, 2 m-1,2 m)$-Latin trade from Theorem 16, yields idempotent $\left(\mu, k^{\prime}, 2 m\right)$ Latin trades for $k^{\prime} \in \Gamma=\Gamma_{1} \cup \Gamma_{2}$, where $\Gamma_{1}=\{0\} \cup\{y, y+1, \ldots, m-1\}$ and $\Gamma_{2}=$ $\{2 y, 2 y+2, \ldots, 2 m-2\} \cup\{2 m-1\}$. Applying Theorem 14 with $l=3$, using the above idempotent $\left(\mu, k^{\prime}, 2 m\right)$-Latin trades with $k^{\prime} \in \Gamma$ along with $(\mu, 2 m, 2 m)$-Latin trades and $(\mu, m, 2 m)$-Latin trades that exist by Theorem 11 and Theorem 12, yields idempotent $\left(\mu, k^{\prime \prime}, 6 m\right)$-Latin trades for each $k^{\prime \prime} \in\left\{\sum_{i=1}^{3} y_{i} \mid y_{1} \in \Gamma\right.$ and $\left.y_{2}, y_{3} \in \Gamma \cup\{m, 2 m\}\right\}$.

It holds that $\left\{\sum_{i=1}^{3} y_{i} \mid y_{1} \in \Gamma_{2}\right.$ and $\left.y_{2}, y_{3} \in \Gamma_{2} \cup\{2 m\}\right\}=\{6 y, 6 y+2, \ldots, 4 y+2 m-$ $4,4 y+2 m-2\} \cup\{4 y+2 m-1,4 y+2 m, \ldots, 6 m-1\}$, and also that $\left\{\sum_{i=1}^{3} y_{i} \mid y_{1} \in\right.$ $\Gamma_{1}$ and $\left.y_{2}, y_{3} \in \Gamma_{1} \cup\{m\}\right\}=\{0\} \cup\{y, y+1, \ldots, m\} \cup\{2 y, 2 y+1, \ldots, 2 m\} \cup\{3 y, 3 y+$ $1, \ldots, 3 m-1\}=\{0\} \cup\{y, y+1, \ldots, 3 m-1\}$ as $y \leqslant m / 4$ implies both $m \geqslant 2 y$ and $2 m \geqslant 3 y$. Then it holds that $\left\{\sum_{i=1}^{3} y_{i} \mid y_{1} \in \Gamma\right.$ and $\left.y_{2}, y_{3} \in \Gamma \cup\{m, 2 m\}\right\} \supseteq\{0\} \cup\{y, y+$ $1, \ldots, 3 m-1\} \cup\{4 y+2 m-1, \ldots, 6 m-1\}=\{0\} \cup\{y, \ldots, 6 m-1\}$, as $m \geqslant 4 y$, and so the proof is complete.

There does not exist a pair of orthogonal Latin squares of order 2, so we do not have a similar result to this lemma when $n=2$. This leaves us with two cases that are of particular interest, as they are not covered by Lemma 23: $m=p$ and $m=2 p$, for $p$ a prime. The next two subsection contain constructions that will be used to fill the spectrum $\mathcal{I S}_{m}^{3}$ for certain $m=p$ and $m=2 p$.

\subsection{Packing construction}

The following theorem uses $\mu$-way Latin trades of order $\lambda$ and volume $s$ in the construction of $(\mu, s, m)$-Latin trades, for certain integers $m \geqslant \lambda^{2}+2 \lambda+1$. Afterwards we will modify the resulting structures to yield idempotent $(\mu, s, m)$-Latin trades. The 3 -way intersection problem for Latin squares has been studied previously, and this will yield the 3-way Latin trades we need in order to apply this construction, which we detail later.

Theorem 24. Suppose there exists a $\mu$-way Latin trade of volume $s$ and of order $\lambda$. For every $m=\lambda(\lambda+a)+b$, where $0<b<\lambda, a \geqslant b+1$, and $\operatorname{gcd}(m, \lambda)=\operatorname{gcd}(\lambda, b)=1$, there exists a $(\mu, s, m)$-Latin trade.

In order to prove this theorem, we construct the $(\mu, s, m)$-Latin trade as follows.

Construction 25. Suppose there exists a $\mu$-way Latin trade $\mathcal{U}=\left(U_{1}, \ldots, U_{\mu}\right)$ of volume $s$, order $\lambda$, and using symbol set $\Omega=[\lambda]$. Let $m=\lambda(\lambda+a)+b$, where $a$ and $b$ are integers with $0<b<\lambda, a \geqslant b+1$, and $\operatorname{gcd}(m, \lambda)=\operatorname{gcd}(\lambda, b)=1$. Let $U_{\alpha}[f]$ be the array obtained from $U_{\alpha}$ by replacing each occurrence of symbol $i$ with symbol $b_{i}+f(\bmod m)$, where $b_{i}=i(\lambda+a-1)$ for each $1 \leqslant i \leqslant \lambda, \alpha \in[\mu]$, and $0 \leqslant f<m$.

We construct $R_{\alpha}$, an $m \times m$ array of cells for each $\alpha \in[\mu]$. For each $f \in\{0, \ldots, m-1\}$, consider the $\lambda \times \lambda$ block of cells within $R_{\alpha}$ given by $B_{f}=\{(i, j) \mid \lambda f<i \leqslant \lambda(f+1), f<$ $j \leqslant f+\lambda$, with $i, j$ taken $\bmod m$, but 0 is identified with $m$ such that $1 \leqslant i, j \leqslant m$. This 


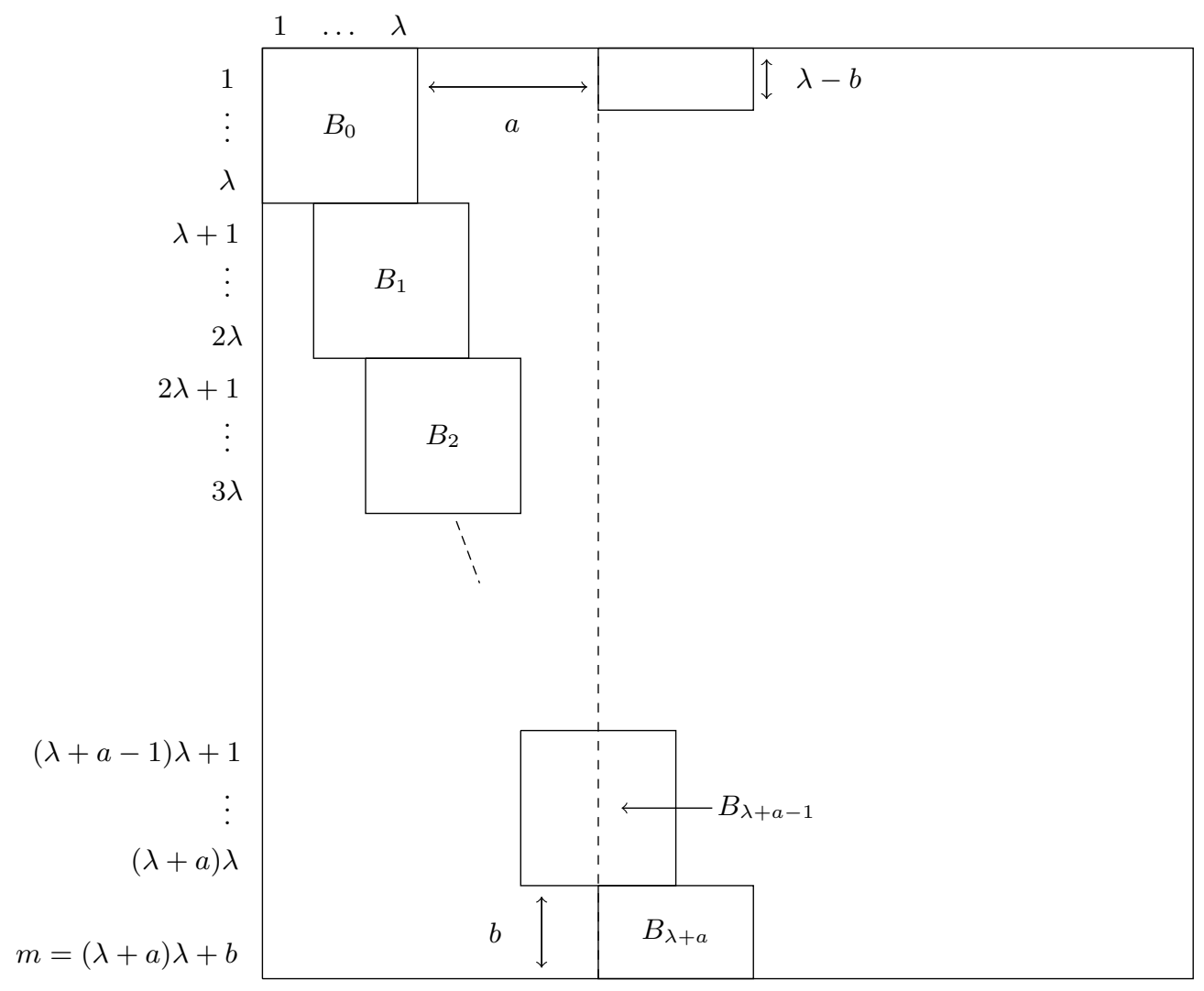

Figure 1: An illustrative example of the placement of blocks in Construction 25

placement of the blocks of cells in $R_{\alpha}$ is displayed visually in Figure 1. Notice $B_{f} \cap B_{g}=\varnothing$ for each $0 \leqslant f, g<m, f \neq g$. Place $U_{\alpha}[f]$ into the cells $B_{f}$ of $R_{\alpha}$, for each $0 \leqslant f<m$, and leave every other cell empty. Then cell $\left(\lambda f+i^{\prime}, f+j^{\prime}\right) \in B_{f}$ is filled in $R_{\alpha}$ with the symbol $U_{\alpha}[f]\left(i^{\prime}, j^{\prime}\right)$, for $1 \leqslant i^{\prime}, j^{\prime} \leqslant \lambda$.

The following proof verifies that the $R_{\alpha}$ form a $(\mu, s, m)$-Latin trade.

Proof. We will show that the collection of $\mu m \times m$ arrays $\mathcal{R}=\left(R_{1}, \ldots, R_{\mu}\right)$ defined by Construction 25 form a $(\mu, s, m)$-Latin trade. We begin by showing that $\mathcal{R}$ forms a $\mu$-way Latin trade of order $m$. This amounts to showing that each $R_{\alpha}$ forms a partial Latin square, as then by construction it is clear that the $\mu$ partial Latin squares form a $\mu$-way Latin trade of order $m$. To this end, we must verify that any symbol appears in a column of $R_{\alpha}$ at most once, and that any symbol appears in a row of $R_{\alpha}$ at most once.

To show the symbols that appear in a column of $R_{\alpha}$ are distinct, we will consider a specific symbol $b_{\lambda}=\lambda(\lambda+a-1)$. This symbol appears only in the block of cells $B_{j(\lambda+a-1)}$ of $R_{\alpha}$, for $0 \leqslant j \leqslant \lambda-1$. The columns of the block of cells $B_{j(\lambda+a-1)}$ of $R_{\alpha}$ are exactly those columns $c$ with $j(\lambda+a-1)<c \leqslant j(\lambda+a-1)+\lambda$. For $0 \leqslant j \leqslant \lambda-1$, the sets of integers $\{j(\lambda+a-1)+1, \ldots, j(\lambda+a-1)+\lambda\}$ are each disjoint, and in the range 
1 to $m$. That is to say that two distinct block of cells $B_{f}$ and $B_{f^{\prime}}$ containing $b_{\lambda}$ do not intersect column-wise, and as a column within each block of cells $B_{f}$ of $R_{\alpha}$ can contain $b_{\lambda}$ at most once, we can conclude that $b_{\lambda}$ appears in each column of $R_{\alpha}$ at most once. By construction, $\left(r, c, b_{\lambda}\right) \in R_{\alpha}$ if and only if $\left(r+\lambda, c+1, b_{\lambda}+1\right) \in R_{\alpha}$, and so symbol $b_{\lambda}+1$ appears in each column of $R_{\alpha}$ at most once. Repeating this argument, we see that every symbol will appear in each column of $R_{\alpha}$ at most once.

To show the symbols that appear in a row of $R_{\alpha}$ are distinct, we consider a specific symbol $a-b-1=b_{\lambda}+(\lambda+a-1)-m$, which we denote as $b_{\lambda+1}$. This symbol appears in the block of cells $B_{j(\lambda+a-1)}$ of $R_{\alpha}$, for $1 \leqslant j \leqslant \lambda$. The rows of the subsquare $B_{j(\lambda+a-1)}$ of $R_{\alpha}$ are exactly those rows $r$ with $j \lambda(\lambda+a-1)<r \leqslant j \lambda(\lambda+a-1)+\lambda$, or equivalently $m-j(\lambda+b)<r \leqslant m-j(\lambda+b)+\lambda$ once we consider $r$ to be taken modulo $m$. For $1 \leqslant j \leqslant \lambda$, the sets of integers $\{m-j(\lambda+b)+1, \ldots, m-j(\lambda+b)+\lambda\}$ are each disjoint. As $\lambda(\lambda+b)<\lambda(\lambda+a)<m$, these sets of integers only contain values in the range 1 to $m$. That is to say that two distinct blocks of cells $B_{f}$ and $B_{f^{\prime}}$ that both contain $b_{\lambda+1}$ do not intersect row-wise, so we can conclude that $b_{\lambda+1}$ appears in each row of $R_{\alpha}$ at most once. By construction, $\left(r, c, b_{\lambda+1}\right) \in R_{\alpha}$ if and only if $\left(r+\lambda, c+1, b_{\lambda+1}+1\right) \in R_{\alpha}$, and so $b_{\lambda+1}+1$ appears in each row of $R_{\alpha}$ at most once. Repeating this argument, we see that every symbol will appear in each row of $R_{\alpha}$ at most once. We have now shown that the $R_{\alpha}$ form a $\mu$-way Latin trade of order $m$.

Now we show that $R_{\alpha}$ is $s$-homogeneous, for each $\alpha \in[\mu]$. For $\alpha \in[\mu]$, the construction filled each of the $m$ blocks $B_{f}$ of $R_{\alpha}$ with $s$ cells, for $0 \leqslant f<m$. As no overlap occurs between the blocks $B_{f}, R_{\alpha}$ was filled by precisely $s m$ filled cells. By construction, if cell $(r, c)$ is filled in $R_{\alpha}$, then it holds that $(r, c, e) \in R_{\alpha}$ if and only if $(r+\lambda, c+1, e+1) \in R_{\alpha}$. Then row $r$ (column $c$, symbol $e$ ) must contain the same number of filled cells as row $r+\lambda$ (column $c+1$, symbol $e+1$ ). We can repeat this argument $m-1$ times to show each row and column will have the same number of filled cells, and that each each symbol will have the same number of occurrences in $R_{\alpha}$ (for the row case, we have used the assumption that $\operatorname{gcd}(m, \lambda)=1)$. Then this implies the $s m$ filled cells are spread evenly amongst the $m$ rows, columns, and symbols. This gives $s$ filled cells per row, $s$ filled cells per column, and $s$ occurrences per symbol.

This shows that each $R_{\alpha}$ is $s$-homogeneous, and so the proof is complete.

Example 26. We demonstrate this technique using a 2-way Latin trade of volume $s=7$ and of order $\lambda=3$ given by the pair of partial Latin squares:

\begin{tabular}{|l|l|l|}
\hline 1 & 2 & 3 \\
\hline 3 & & 1 \\
\hline 2 & 3 & \\
\hline
\end{tabular}

\begin{tabular}{|l|l|l|}
\hline 2 & 3 & 1 \\
\hline 1 & & 3 \\
\hline 3 & 2 & \\
\hline
\end{tabular}

We will take $a=2$ and $b=1$, giving the order of the resulting trade as $\lambda(\lambda+a)+b=16$. Then $b_{1}=4, b_{2}=8$, and $b_{3}=12$. Using the first of the above partial Latin squares of order 3 and volume 7 in the construction gives a partial Latin square of order 16 that is 7-homogeneous: 


\begin{tabular}{|c|c|c|c|c|c|c|c|c|c|c|c|c|c|c|c|}
\hline 4 & 8 & 12 & & & 1 & & 9 & & & 2 & 6 & & & & \\
\hline 12 & & 4 & & & 13 & 1 & & & & & 15 & 3 & 7 & & \\
\hline 8 & 12 & & & & & 10 & 14 & 2 & & & 7 & & 15 & & \\
\hline & 5 & 9 & 13 & & & 2 & & 10 & & & 3 & 7 & & & \\
\hline & 13 & & 5 & & & 14 & 2 & & & & & 16 & 4 & 8 & \\
\hline & 9 & 13 & & & & & 11 & 15 & 3 & & & 8 & & 16 & \\
\hline & & 6 & 10 & 14 & & & 3 & & 11 & & & 4 & 8 & & \\
\hline & & 14 & & 6 & & & 15 & 3 & & & & & 1 & 5 & 9 \\
\hline & & 10 & 14 & & & & & 12 & 16 & 4 & & & 9 & & 1 \\
\hline & & & 7 & 11 & 15 & & & 4 & & 12 & & & 5 & 9 & \\
\hline 10 & & & 15 & & 7 & & & 16 & 4 & & & & & 2 & 6 \\
\hline 2 & & & 11 & 15 & & & & & 13 & 1 & 5 & & & 10 & \\
\hline & & & & 8 & 12 & 16 & & & 5 & & 13 & & & 6 & 10 \\
\hline 7 & 11 & & & 16 & & 8 & & & 1 & 5 & & & & & 3 \\
\hline & 3 & & & 12 & 16 & & & & & 14 & 2 & 6 & & & 11 \\
\hline 11 & & & & & 9 & 13 & 1 & & & 6 & & 14 & & & 7 \\
\hline
\end{tabular}

We can use the second partial Latin square of order 3 and volume 7 to construct a similar partial Latin square of order 16 that is 7-homogeneous. Together, these partial Latin squares form a 2-way 7-homogeneous Latin trade of order 16.

Theorem 27. Suppose there exists a $\mu$-way Latin trade of volume $s$ and of order $\lambda$, with $\lambda \geqslant 1$. For every $m=\lambda(\lambda+a)+b$, where $0<b<\lambda$, $a \geqslant b+1$, and $\operatorname{gcd}(m, \lambda)=$ $\operatorname{gcd}(\lambda, b)=1$, there exists a circulant idempotent $(\mu, s, m)$-Latin trade.

Proof. Consider the $\mu$ arrays $R_{1}, \ldots, R_{\mu}$ from Construction 25 . Define the array $\bar{R}_{\alpha}$ by the set of ordered triples $\bar{R}_{\alpha}=\left\{\left(\sigma_{1}(r), c, \sigma_{2}(e)\right) \mid(r, c, e) \in R_{\alpha}\right\}$ with $\sigma_{1}(r)=\lambda^{-1} \cdot(r-1)-1$ $(\bmod m)$ and $\sigma_{2}(e)=e-2(\lambda+a-1)(\bmod m)$, for each $\alpha \in[\mu]$, where $\lambda^{-1}$ is the unique inverse of $\lambda(\bmod m)$ which exists by the assumption that $\operatorname{gcd}(m, \lambda)=1$. Both $\sigma_{1}$ and $\sigma_{2}$ are permutations of $[m]$. As the $R_{\alpha}$ form a $(\mu, s, m)$-Latin trade, the $\bar{R}_{\alpha}$ also form a $(\mu, s, m)$-Latin trade as the three properties of Definition 1 are invariant under permutation swaps of the rows, columns, and symbols.

Since $(r, c, e) \in R_{\alpha}$ implies $(r+\lambda, c+1, e+1) \in R_{\alpha}$ by construction, it follows that $(r, c, e) \in \bar{R}_{\alpha}$ implies $(r+1, c+1, e+1) \in \bar{R}_{\alpha}$, and so $\bar{R}_{\alpha}$ is a circulant $(\mu, s, m)$-Latin trade.

We show that $1 \notin \mathcal{R}_{1}\left(\bar{R}_{\alpha}\right), 1 \notin \mathcal{C}_{1}\left(\bar{R}_{\alpha}\right)$, and $(1,1) \notin \mathcal{S}\left(\bar{R}_{\alpha}\right)$. Noting that $\sigma_{1}^{-1}(1)=$ $2 \lambda+1$ and $\sigma_{2}^{-1}(1)=2 \lambda+2 a-1$, this is equivalent to showing $2 \lambda+2 a-1 \notin \mathcal{R}_{2 \lambda+1}\left(R_{\alpha}\right)$, $2 \lambda+2 a-1 \notin \mathcal{C}_{1}\left(R_{\alpha}\right)$, and $(2 \lambda+1,1) \notin \mathcal{S}\left(R_{\alpha}\right)$.

We first show that $2 \lambda+2 a-1 \notin \mathcal{R}_{2 \lambda+1}\left(R_{\alpha}\right)$. The symbol $2 \lambda+2 a-1$ appears only in the blocks $B_{j(\lambda+a-1)+b+3 \lambda+2 a-1}$ of $R_{\alpha}$ for $0 \leqslant j \leqslant \lambda-1$, hence it only appears within the rows $T=\cup_{j=0}^{\lambda-1}\{\lambda(j(\lambda+a-1)+b+3 \lambda+2 a-1)+1, \ldots, \lambda(j(\lambda+a-1)+b+3 \lambda+2 a-1)+\lambda\}$. If we perform a change in variables, sending $j$ to $\lambda-2-j$, then $T=\cup_{j=0}^{\lambda-2}\{\lambda+1+j(\lambda+$ $b), \ldots, 2 \lambda+j(\lambda+b)\} \cup\{m-b+1, \ldots, m\} \cup\{1, \ldots, \lambda-b\}$, which does not contain $2 \lambda+1$. Then the symbol $2 \lambda+2 a-1$ does not appear in the row $2 \lambda+1$. 
Secondly we show that $2 \lambda+2 a-1 \notin \mathcal{C}_{1}\left(R_{\alpha}\right)$. The symbol $2 \lambda+2 a-1$ appears exactly in the blocks $B_{j(\lambda+a-1)+b+3 \lambda+2 a-1}$ of $R_{\alpha}$, for $0 \leqslant j \leqslant \lambda-1$. These blocks only use the columns $\cup_{j=0}^{\lambda-1}\{j(\lambda+a-1)+b+3 \lambda+2 a, \ldots, j(\lambda+a-1)+b+3 \lambda+2 a+\lambda-1\}=\cup_{j=0}^{\lambda-3}\{j(\lambda+a-1)+$ $b+3 \lambda+2 a, \ldots, j(\lambda+a-1)+b+3 \lambda+2 a+\lambda-1\} \cup\{2, \ldots, \lambda+1\} \cup\{\lambda+a+1, \ldots, \lambda+a+\lambda\}$. As such, the column with index 1 does not contain $2 \lambda+2 a-1$.

Thirdly we show that $(2 \lambda+1,1) \notin \mathcal{S}\left(R_{\alpha}\right)$. Suppose for the sake of contradiction that $(2 \lambda+1,1) \in \mathcal{S}\left(R_{\alpha}\right)$. Then there must be some block $B_{f^{\prime}}$ that contains cell $(2 \lambda+1,1)$, $0 \leqslant f^{\prime} \leqslant m-1$. As $B_{1}=\{(i, j) \mid \lambda+1 \leqslant i \leqslant 2 \lambda, 2 \leqslant j \leqslant 1+\lambda\}, B_{f^{\prime}}$ cannot contain the cell $(2 \lambda, 2)$. Then $B_{f^{\prime}}$ must either have exactly the rows $\{2 \lambda+1, \ldots, 3 \lambda\}$, or have exactly the columns $\{m-\lambda+2, \ldots, m\} \cup\{1\}$.

The former implies $B_{f^{\prime}}$ contains exactly the same rows as $B_{2}$. As the first rows are the same, $f^{\prime} \lambda+1 \equiv 2 \lambda+1 \bmod m$, and as $\operatorname{gcd}(\lambda, m)=1$, we have $f^{\prime}=2$. But then $B_{2}$ must contain cell $(2 \lambda+1,1)$, which when we look at the columns implies $\lambda+2 \geqslant m+1$. As $m \geqslant \lambda(\lambda+2)+1$ and $\lambda \geqslant 1$, this is impossible.

The later implies $B_{f^{\prime}}=B_{1-\lambda}=B_{m+1-\lambda}$. Then each of the $m$ rows are represented at least once in the set of $\lambda+1$ blocks $\left\{B_{m+1-\lambda}, B_{m+2-\lambda}, \ldots, B_{m-1}\right\} \cup\left\{B_{0}, B_{1}\right\}$, but these $\lambda+1$ blocks only use $\lambda$ rows each, and so $\lambda(\lambda+1)$ rows in total. This implies $m \leqslant \lambda(\lambda+1)$, which contradicts the fact that $m=\lambda(\lambda+a)+b$ and that $a>b \geqslant 1$.

Then none of the cases are possible, forming a contradiction, and so $(2 \lambda+1,1) \notin \mathcal{S}\left(R_{\alpha}\right)$. This completes the proof.

The three-way intersection problem for Latin squares has been studied in [1], where the authors consider three Latin squares $L_{1}, L_{2}, L_{3}$ of order $n$ with common intersection $P=$ $L_{1} \cap L_{2}=L_{1} \cap L_{3}=L_{2} \cap L_{3}$. The collection of partial Latin squares $\left(L_{1} \backslash P, L_{2} \backslash P, L_{3} \backslash P\right)$ forms a 3-way Latin trade of volume $n^{2}-|P|$ and of any order $n^{\prime}$ greater than or equal to $n$ (as we allow empty rows and columns in 3-way Latin trades). We can thus interpret the results of [1] in terms of 3 -way Latin trades and combine them with Theorem 27 to yield:

Theorem 28. For $\lambda \geqslant 3$, there exists circulant idempotent $(3, k, m)$-Latin trades for $m=\lambda(\lambda+a)+b$, where $0<b<\lambda, \operatorname{gcd}(\lambda, b)=1$, and $a \geqslant b+1$, and:

- $k \in\{0,9\}$, for $\lambda=3$;

- $k \in\{0,9,12,15,16\}$, for $\lambda=4$;

- $k \in\{0,9,12,15,16\}$ or $18 \leqslant k \leqslant 25$, for $\lambda=5$;

- $k \in\{0,9,12\}$ or $15 \leqslant k \leqslant \lambda^{2}$, for $\lambda \geqslant 6$.

\subsection{Construction via $R P B D$ s}

Definition 29. A $(v, M, \lambda)$ pairwise balanced design, denoted $P B D(v, M, \lambda)$, is a pair $(V, B)$, with $V$ a set of $v$ symbols and $B$ a set of subsets of $V$ (each subset is called a block) with sizes from $M$, such that each pair of elements of $V$ can be found in exactly $\lambda$ blocks of $B$. 
Definition 30. A resolvable $(v, M, \lambda, n)$ pairwise balanced design, which we denote by $R P B D(v, M, \lambda, n)$, is a pair $(V, B)$ along with $n$ resolution classes $R_{1}, \ldots, R_{n}$, such that $(V, B)$ is a $P B D(v, M, \lambda)$, the sets $R_{1}, \ldots, R_{n}$ partition $B$, and each symbol appears in precisely one block of each resolution class.

Theorem 31. Suppose there exists a $R P B D(v, M, 1, n+1),(V, B)$, with resolution classes $R_{1}, \ldots, R_{n}$ and $R_{\infty}$. Suppose there exists integers $d_{i} \geqslant 1,1 \leqslant i \leqslant n$, such that for each $b \in R_{i}$ there exists an idempotent $\left(\mu,|b|-d_{i},|b|\right)$-Latin trade and integer $d_{\infty} \geqslant 0$ such that for each $b \in R_{\infty}$ there exists $a\left(\mu,|b|-d_{\infty},|b|\right)$-Latin trade. Then there exists a $\left(\mu, v+n-\sum_{i=1}^{n} d_{i}-d_{\infty}, v\right)$-Latin trade.

The following construction was suggested by Prof. L. Zhu, which is a modification of a construction for sets of idempotent Latin squares (see [18], page 188). After the construction, we will give a proof to show that the construction yields Theorem 31 .

Construction 32. Take $(V, B)$, a $R P B D(v, M, 1, n+1)$ with resolution classes $R_{1}, \ldots, R_{n}$ and $R_{\infty}$. Suppose there are integers $d_{i} \geqslant 1,1 \leqslant i \leqslant n$, such that for each $b \in R_{i}$ there exists an idempotent $\left(\mu,|b|-d_{i},|b|\right)$-Latin trade, and integer $d_{\infty} \geqslant 0$ such that for each $b \in R_{\infty}$ there exists a $\left(\mu,|b|-d_{\infty},|b|\right)$-Latin trade. We impose an arbitrary total ordering $<$ on $V$. For a block $b \subseteq V$, define $b^{h}$ to be the $h$ th smallest symbol in $b$ when $b$ is considered under the ordering imposed on $V$, for $1 \leqslant h \leqslant|b|$. That is $\left\{b^{1}, \ldots, b^{|b|}\right\}=b$ and $b^{i}<b^{i+1}$ for $1 \leqslant i \leqslant|b|-1$.

We construct $\mu v \times v$ arrays, $T_{1}, \ldots, T_{\mu}$, each with rows and columns indexed by $V$. For each $1 \leqslant i \leqslant n$ and each block $b \in R_{i}$, let $\mathcal{S}=\left(S_{1}, \ldots, S_{\mu}\right)$ be an idempotent $\left(\mu,|b|-d_{i},|b|\right)$-Latin trade on the set of symbols $\Omega=[|b|]$. For each block $b \in R_{\infty}$, let $\mathcal{S}=\left(S_{1}, \ldots, S_{\mu}\right)$ be a $\left(\mu,|b|-d_{\infty},|b|\right)$-Latin trade on the set of symbols $\Omega=[|b|]$. Whenever $(r, c, e) \in S_{\alpha}$ put $\left(b^{r}, b^{c}, b^{e}\right)$ into $T_{\alpha}$, for $1 \leqslant r, c \leqslant|b|$. Note that if $(r, c)$ is empty in $S_{\alpha}$ for any $1 \leqslant r, c \leqslant|b|$, then $\left(b^{r}, b^{c}\right)$ is left empty in $T_{\alpha}$.

Proof. Consider the $\mu v \times v$ arrays $\mathcal{T}=\left(T_{1}, \ldots, T_{\mu}\right)$ from Construction 32. We will show that $\mathcal{T}$ is a $\left(\mu, v+n-\sum_{i=1}^{n} d_{i}-d_{\infty}, v\right)$-Latin trade.

During the construction, any single cell $(r, c, e) \in T_{\alpha}$ with $|\{r, c, e\}| \leqslant 2$ must have been constructed using some $x \in R_{\infty}$, as the other blocks were replaced during the construction by idempotent $(\mu, k, m)$-Latin trades, which would imply $|\{r, c, e\}|=3$ by the definition of idempotent $\mu$-way Latin trades.

Suppose the construction filled two cells $\left(r_{1}, c_{1}, e_{1}\right)$ and $\left(r_{2}, c_{2}, e_{2}\right)$ of $T_{\alpha}$ such that the two cells have two of three indices the same. Let $a$ and $b$ be the values of the two identical indices (for example if the two cells we are observing are $\left(r, c, e_{1}\right),\left(r, c, e_{2}\right) \in T_{\alpha}$ with $e_{1} \neq$ $e_{2}$, then $a=r$ and $b=c$ ). If distinct blocks $x$ and $y$ were used respectively to construct $\left(r_{1}, c_{1}, e_{1}\right)$ and $\left(r_{2}, c_{2}, e_{2}\right)$, then $\{a, b\} \subseteq x \cap y$. By the definition of a $\operatorname{PBD}(v, M, 1)$, $|x \cap y| \leqslant 1$, and so $a=b$. Then $\left|\left\{r_{1}, c_{1}, e_{1}\right\}\right| \leqslant 2$ and $\left|\left\{r_{2}, c_{2}, e_{2}\right\}\right| \leqslant 2$, and so $x, y \in R_{\infty}$, which implies $|x \cap y|=0$ as $x$ and $y$ are distinct blocks in the same resolution class. This forms a contradiction, as $a \in x \cap y$. So any two filled cells that have two of three indices the same were both filled during construction using the same block. 
As we filled $\mu$-way Latin trades into $T_{\alpha}$ from these blocks, it follows that no cell was filled twice, each row contains each symbol at most once, and each column contains each symbol at most once. Then each $T_{\alpha}$ is a partial Latin square.

To see $\mathcal{T}$ forms a $\mu$-way Latin trade, it is enough to note that $\mathcal{S}\left(T_{\alpha}\right)$ must be the same for each $\alpha \in[\mu]$; that each filled cell $(r, c)$ was filled differently in each $T_{\alpha}, \alpha \in[\mu]$; and that each row (resp. column) contains setwise the same symbols, each of which are clear from the construction. Then the $\mu$ arrays $T_{\alpha}$ form a $\mu$-way Latin trade of order $v$.

We are left to show $\mathcal{T}$ is $\left(v+n-\sum_{i=1}^{n} d_{i}-d_{\infty}\right)$-homogeneous. To show that there are $v+n-\sum_{i=1}^{n} d_{i}-d_{\infty}$ filled cells in each row, observe that for each symbol $r$ and each resolution class $R_{i}$, there is precisely one block $b_{i}$ such that $r \in b_{i} \in R_{i}$, for each $i$ with $1 \leqslant i \leqslant n$ or $i=\infty$. Then any filled cells in row $r$ are in the cells $b_{i} \times b_{i}$ for some $i$ with $1 \leqslant i \leqslant n$ or $i=\infty$. There are $\left|b_{i}\right|-d_{i}$ filled cells in the intersection of row $r$ and the block of cells $b_{i} \times b_{i}$, for $1 \leqslant i \leqslant n$ and $i=\infty$, showing row $r$ has a total of $\sum_{i=1}^{n}\left(\left|b_{i}\right|-d_{i}\right)+\left(\left|b_{\infty}\right|-d_{\infty}\right)=v+n-\sum_{i=1}^{n} d_{i}-d_{\infty}$ filled cells. The proof is analogous for the number of filled cells per column and for the number of occurrences of each symbol. This shows $\mathcal{T}$ is a $\mu$-way Latin trade of order $v$ that is $\left(v+n-\sum_{i=1}^{n} d_{i}-d_{\infty}\right)$-homogeneous, so we are done.

Theorem 33. Suppose there exists a $R P B D(v, M, 1, n+1),(V, B)$ with resolution classes $R_{1}, \ldots, R_{n}$ and $R_{\infty}$. Suppose there exists integers $d_{i} \geqslant 1,1 \leqslant i \leqslant n$, such that for each $b \in R_{i}$ there exists an idempotent $\left(\mu,|b|-d_{i},|b|\right)$-Latin trade and integer $d_{\infty} \geqslant 1$ such that for each $b \in R_{\infty}$ there exists an idempotent $\left(\mu,|b|-d_{\infty},|b|\right)$-Latin trade. Then there exists an idempotent $\left(\mu, v+n-\sum_{i=1}^{n} d_{i}-d_{\infty}, v\right)$-Latin trade.

Proof. Consider the $\mu v \times v$ arrays $\mathcal{T}=\left(T_{1}, \ldots, T_{\mu}\right)$ from Construction 32 using idempotent $\left(\mu,|b|-d_{i},|b|\right)$-Latin trades to fill in the squares $b \times b$, for blocks $b \in R_{\infty}$.

The proof of Theorem 31 shows $\mathcal{T}$ is a $\left(\mu, v+n-\sum_{i=1}^{n} d_{i}-d_{\infty}, v\right)$-Latin trade. We show that $\mathcal{T}$ is idempotent. Assume for the sake of contradiction that $\mathcal{T}$ is not idempotent. Then there exists a $(r, c, e) \in T_{\alpha}$ with at least two of the three indices $r, c, e$ the same. This only occurs when the block $x$ with $\{r, c, e\} \subseteq x$ and $x \in R_{i}$ was used along with a non-idempotent $(\mu, k,|x|)$-Latin trade to construct the cell $(r, c, e) \in T_{\alpha}$. But there is no such $x$ as each of the $(\mu, k,|x|)$-Latin trades are idempotent. Then $\mathcal{T}$ is idempotent.

We wish to choose an $\operatorname{RPBD}(v, M, 1, n+1)$ with resolution classes $R_{1}, \ldots, R_{n}$ and $R_{\infty}$ such that there will exist idempotent $\left(\mu,|b|-d_{i},|b|\right)$-Latin trades for each $b \in R_{i}$, for some $d_{i} \geqslant 1$ and $i \in\{1, \ldots, n, \infty\}$. By making $M$ contain as few values as possible, we can limit the number of idempotent $(\mu, k, m)$-Latin trades that are required to exist, as $|b| \in M$. A resolvable transversal design is a $R P B D(\alpha n,\{\alpha, n\}, 1, n+1)$, and so suits our purposes as $|M| \leqslant 2$. We are able to modify the resolvable transversal design by removing elements in order to yield $R P B D(v, M, 1, n+1)$ such that $v$ can be any positive integer, while $M$ contains as few values as possible.

Definition 34. A transversal design $T D(\alpha, n)$ of order $n$ and block size $\alpha$, is a triple $(V, G, B)$ such that: 
1. $V$ is a set of $\alpha n$ elements;

2. $G$ is a partition of $V$ into $\alpha$ subsets (called the groups), each of size $n$;

3. $B$ is a collection of subsets of $V$ (called the blocks), each of size $\alpha$; and

4. every unordered pair of elements of $V$ appears in precisely one block of $B$, or one group of $G$, but not both.

Definition 35. A resolvable transversal design $R T D(\alpha, n)$ of order $n$ and block size $\alpha$, is a triple $(V, G, B)$ such that $B$ can be partitioned into $n$ resolution classes $R_{1}, \ldots, R_{n}$, such that each $R_{i}$ is a partition of $V$ into $n$ classes.

The following two lemmata are well known (See III.3.2 and III.3.3 in [11]).

Lemma 36. A RTD $(\alpha, n)$ is equivalent to a $T D(\alpha+1, n)$.

Lemma 37. For $n$ a prime power and $\alpha \leqslant n$, there exists a $T D(\alpha+1, n)$ and hence there exists a $R T D(\alpha, n)$.

Construction 38. Consider a $R T D(\alpha, n)(V, G, B)$ with resolution classes $R_{1}, \ldots, R_{n}$, and let $G=\left\{G_{1}, \ldots, G_{\alpha}\right\}$. We take $0 \leqslant x \leqslant n, 0 \leqslant \gamma \leqslant \alpha$ and $0 \leqslant u \leqslant n-x$. We will form a $R P B D(v, M, 1, n+1),(\hat{V}, \hat{B})$, by deleting a set of $(n-x) \gamma+u$ points, which we label as $\bar{V}$. The points $\bar{V}$ that we delete will be $n-x$ points from $G_{i}$ for each $i$ with $\alpha-\gamma+1 \leqslant i \leqslant \alpha$, and $u$ points of $G_{\alpha-\gamma}$. Each point that was removed from a group is also removed from any block that contains it. This gives point set $\hat{V}=V \backslash \bar{V}$, block set $\hat{B}=\{b \backslash \bar{V} \mid b \in G \cup B\}$, and $n+1$ resolution classes $\hat{R}_{i}=\left\{b \backslash \bar{V} \mid b \in R_{i}\right\}$ for $1 \leqslant i \leqslant n$ and $\hat{R}_{\infty}=\{b \backslash \bar{V} \mid b \in G\}$.

This results in a $R P B D(n \alpha-n \gamma+x \gamma-u, M, 1, n+1)$ with $M=\{\alpha-(\gamma+1), \ldots, \alpha\} \cup$ $\{x, n-u, n\}$.

It will be useful to summarize the results of this section, which yield the following lemma:

Lemma 39. Take $n$ a prime power and positive integers $\alpha, x, \gamma$, and $u$ such that $\alpha \leqslant n$, $0 \leqslant x \leqslant n, 0 \leqslant \gamma \leqslant \alpha$, and $0 \leqslant u \leqslant n-x$. Suppose there exists integers $d_{i} \geqslant 1$, $1 \leqslant i \leqslant n$ and $d_{\infty} \geqslant 1$, such that for each $b$ with $\alpha-(\gamma+1) \leqslant b \leqslant \alpha$ there exists an idempotent $\left(3, b-d_{i}, b\right)$-Latin trade when $1 \leqslant i \leqslant n$, and for each $b \in\{x, n-u, n\}$ there exists an idempotent $\left(3, b-d_{\infty}, b\right)$-Latin trade. Then there exists an idempotent $\left(3, v+n-\sum_{i=1}^{n} d_{i}-d_{\infty}, v\right)$-Latin trade, where $v=n \alpha-n \gamma+x \gamma-u$.

Proof. For these values of $n, \alpha, x, \gamma$, and $u$, Lemma 37 gives us a $R T D(\alpha, n)$, which we can use in Construction 38 to yield a resolvable pairwise balanced design that can be used in Theorem 33 along with the given idempotent $\left(3, b-d_{i}, b\right)$-Latin trades that have been assumed to exist, for $i \in\{1, \ldots, n, \infty\}$, to yield the result. 


\section{Result when $\mu=3$}

We will develop an inductive proof for the existence of idempotent $(3, k, m)$-Latin trades for $m>194$, however we will require the knowledge of the existence of a great deal of base cases. To this end, we will use a computer program to combine the results so far stated in this paper to deduce the spectrum $\mathcal{I S}_{m}^{3}$ for $m \leqslant 2^{18}$. We will create two computer programs, Program A and Program B, which are implemented in $\mathrm{C}++[15]$.

We begin by finding the spectrum $\mathcal{I S}_{m}^{3}$ for $m \leqslant 5618$ using Program A. The value $5618=2 \cdot 53^{2}$ was chosen as there was some difficulty filling in the spectrum $\mathcal{I S}_{5618}^{3}$ later on, stemming from the fact that not enough is known about $\mathcal{I S}_{2.53}^{3}$, and so Lemma 23 cannot be used to fill in the spectrum $\mathcal{I S}_{5618}^{3}$. We split the computation into four parts. Parts 1, 2, and 4 are straightforward to program, but there is some complications with Part 3. We begin with a $(5618+1) \times(5618+1)$ array of booleans $A=\left[a_{k, m}\right]$, where we set $a_{k, m}=$ false for each $0 \leqslant k, m \leqslant 5618$. When we find that there does exist an idempotent $(3, k, m)$-Latin trade, we set $a_{k, m}=$ true.

PART 1: As there trivially exists an idempotent $(3,0, m)$-Latin trade, we set $a_{0, m}=$ true for each $0 \leqslant m \leqslant 5618$. As the existence of idempotent $(3, k, m)$-Latin trades in Theorem 16, Theorem 21, Theorem 22, and Theorem 28 do not depend on the existence of smaller idempotent $\left(3, k^{\prime}, m^{\prime}\right)$-Latin trades, we set $a_{k, m}=$ true for these values.

We can use the idempotent $\left(2, k_{1}, 2 m^{\prime}+1\right)$-Latin trades of Theorem 20 when $3 \leqslant k_{1}<$ $2 m^{\prime}+1,2 m^{\prime}+1 \geqslant 5$, and $\left(k, 2 m^{\prime}+1\right) \neq(3,5)$, along with the $\left(2, k_{2}, m_{2}\right)$-Latin trades of Theorem 10 for $2 \leqslant k_{2} \leqslant m_{2}$, with $k_{i}=2$ only if $m_{i}$ is even, with Theorem 13 to yield an idempotent $\left(3, k_{1} k_{2},\left(2 m^{\prime}+1\right) m_{2}\right)$-Latin trade. This does not depend on the existence of smaller idempotent $(3, k, m)$-Latin trades, so we set $a_{k_{1} k_{2},\left(2 m^{\prime}+1\right) m_{2}}=$ true under these conditions.

PART 2: Theorem 12 and Theorem 14 each require the knowledge of the existence of smaller idempotent $(3, k, m)$-Latin trades, however we can gather this information from what we have stored in $A$. Theorem 14 also uses the existence of non-idempotent $(3, m, m)$ Latin trades, and a non-idempotent $(3,5,6)$-Latin trade (an example of a $(3,5,6)$-Latin trade is shown in the next section).

PART 3: Programming Lemma 39 is not completely straightforward, as the time required can be quite large if not done with due care. We implement Lemma 39 twice. The first implementation uses $d_{\infty}=1$, and the second implementation uses $\gamma=0$. Both of these restrictions speed up the computation immensely, and the values not covered by one are covered by the other. By first looping over $\alpha$ and $\gamma$, we can store the values of $d_{i}$ such that there exists an idempotent $\left(3, b-d_{i}, b\right)$-Latin trade for each $b$ with $\alpha-\gamma-1 \leqslant b \leqslant \alpha$. Then we are able to find the possible values of $\sum_{i=1}^{n} d_{i}$ without much extra computation as we increase $n$, by storing the previously computed values of $\sum_{i=1}^{n-1} d_{i}$.

PART 4: We once again apply the procedure for Theorem 12, which fills in a couple of gaps in the spectrum introduced incidentally in Part 3.

Performing this computation gives the following lemma:

Lemma 40. For $14 \leqslant m \leqslant 5618$, there exists idempotent $(3, k, m)$-Latin trades for $5 \leqslant$ $k \leqslant m$ except, perhaps, for those values in Table 1. 
We need to extend the base results further, which we achieve by way of another computer program, Program B. We begin with an array of $2^{18}+1$ booleans $B=\left[b_{m}\right]$, where we set $b_{m}=$ false for each $0 \leqslant m \leqslant 2^{18}$. When we find that there exists idempotent $(3, k, m)$-Latin trades for every $5 \leqslant k \leqslant m$, we set $b_{m}=$ true. We begin by setting the values of $b_{m}$ to be true when the values $a_{k, m}$ each are true for $5 \leqslant k \leqslant m$. Then Lemma 23 with $y=5$ tells us that we can set $b_{n m}$ to be true whenever $m \geqslant 10, b_{m}$ is true, $n \geqslant 3$, and if $n=6$ then $m \geqslant 20$.

In the case that $m$ is a prime or twice a prime, we can apply Theorem 22 and Theorem 28 to yield idempotent $(3, k, m)$-Latin trades for $5 \leqslant k \leqslant l_{1}$, and we can apply Lemma 39 with $x=7$ to yield idempotent $(3, k, m)$-Latin trades for $l_{2} \leqslant k \leqslant m$, for some integers $l_{1}, l_{2}$. To save computation, we only consider Lemma 39 with $\gamma \in\{0,1\}$ and $d_{\infty}=1$. Note that this means $\left(3, b-d_{\infty}, b\right)$-Latin trades always exist for $b \geqslant 7$, as stated in Theorem 16 . Our program checks if $b_{\alpha-2}=b_{\alpha-1}=b_{\alpha}=$ true for each $\alpha \geqslant 16$ and $n \geqslant \alpha$. In this case, we assume $d_{i} \leqslant \alpha-7$. Then the conditions of a $\left(3, \alpha-(\gamma+1)-d_{i}, \alpha-(\gamma+1)\right)$-Latin trade existing hold independently of whether $\gamma=0$ or $\gamma=1$. Then it will be more convenient to write $u^{\prime}=\gamma(n-7)+u$. If so, for each $m=n \alpha-u^{\prime}$ with $n \geqslant \alpha, 0 \leqslant u^{\prime} \leqslant 2(n-7)$, and $m$ a prime or twice a prime, we know that there exists an idempotent $(3, k, m)$-Latin trade for each $k$ with $m+n-n(\alpha-7)-1=8 n-u^{\prime}-1 \leqslant k \leqslant m-1$. To find the existence of idempotent $(3, k, m)$-Latin trades with $5 \leqslant k<8 n-u^{\prime}-1$, we find the greatest $\lambda$ with $m=\lambda(\lambda+a)+b, a>b, \operatorname{gcd}(m, \lambda)=1$, and $\lambda \geqslant 5$. Then Theorem 28 yields the existence of idempotent $(3, k, m)$-Latin trades with $18 \leqslant k \leqslant \lambda^{2}$. There exists idempotent $(3, k, m)$-Latin trades for $5 \leqslant k \leqslant 17$ by Theorem 22 . If $8 n-u^{\prime}-1 \leqslant \lambda^{2}$, then there exists an idempotent $(3, k, m)$-Latin trade for $5 \leqslant k \leqslant m-1$, and so we set $b_{m}$ to be true.

Performing this computation gives the following lemma:

Lemma 41. For $14 \leqslant m \leqslant 2^{18}$, there exists idempotent $(3, k, m)$-Latin trades for $5 \leqslant$ $k \leqslant m$ except, perhaps, for those values in Table 1.

We have been able to apply Lemma 39 in this computation as we have been able to run a procedure to check which integers $n$ are prime powers. In order to create a theoretic construction, we restrict the prime powers that we use, so that $n$ is of the form $2^{p}$, for an integer $p$. We are then able to show, despite this restriction, that Lemma 39 can yield a large portion of the spectrum of $(3, k, m)$-Latin trades for all $m \geqslant 2^{18}$.

Lemma 42. Take $p \geqslant 10$. Suppose there exists idempotent $\left(3, k^{\prime}, m^{\prime}\right)$-Latin trades for $5 \leqslant k^{\prime} \leqslant m^{\prime}-1$ and $2^{p-2}-6 \leqslant m^{\prime} \leqslant 2^{p}$. Then there exists idempotent $(3, k, m)$-Latin trades for $14 \cdot 2^{p} \leqslant k \leqslant m$ and $2^{2 p-2}<m \leqslant 2^{2 p}$.

Proof. Take $\alpha=n=2^{p}, p \geqslant 10,0 \leqslant \gamma \leqslant \gamma_{\max }, \gamma_{\max }=2^{p}-2^{p-2}+5, x=7$, and $0 \leqslant u \leqslant n-7$. We assume the existence of idempotent $\left(3, k^{\prime}, m^{\prime}\right)$-Latin trades when $5 \leqslant k^{\prime} \leqslant m^{\prime}-1$ and $\alpha-\left(\gamma_{\max }+1\right) \leqslant m^{\prime} \leqslant \alpha$, noting that $\alpha-\left(\gamma_{\max }+1\right)=2^{p-2}-6$. There exists a $\left(3, m^{\prime}-1, m^{\prime}\right)$-Latin trade for $m^{\prime} \in\{7, n-u, n\}$ by Theorem 16, as $m^{\prime} \geqslant 7$.

Then Lemma 39 with these idempotent $\left(3, k^{\prime}, m^{\prime}\right)$-Latin trades yields an idempotent $\left(3, m+n-\sum_{i=1}^{n} d_{i}-d_{\infty}, m\right)$-Latin trade with $m=n \alpha-\gamma(n-7)-u$, where $1 \leqslant d_{i} \leqslant$ $\alpha-(\gamma+1)-5$ for $1 \leqslant i \leqslant n$, and $d_{\infty}=1$. Taking $d_{i} \leqslant \alpha-(\gamma+1)-5$ assures 
us that $m^{\prime}-d_{i} \geqslant 5$ for each $m^{\prime}$ with $\alpha-(\gamma+1) \leqslant m^{\prime} \leqslant \alpha$, and so an idempotent $\left(3, m^{\prime}-d_{i}, m^{\prime}\right)$-Latin trade exists by our assumptions.

Then this procedure yields an idempotent $(3, k, m)$-Latin trade for each $k \in\{m+n-$ $n(\alpha-(\gamma+1)-5)-1, \ldots, m-1\}=\{7 \gamma+7 n-u-1, \ldots, m-1\} \supseteq\{14 n, \ldots, m-1\}$, which holds for each $m=n \alpha-\gamma(n-7)-u$ within $n \alpha-\left(\gamma_{\max }+1\right)(n-7) \leqslant m \leqslant n \alpha$. In particular, it holds that $n \alpha-\left(\gamma_{\max }+1\right)(n-7)=2^{2 p}-\left(2^{p}-2^{p-2}+6\right)\left(2^{p}-7\right)=$ $2^{p-2}-3 \cdot 2^{p-2}+42 \leqslant 2^{2 p-2}$ and so there exists an idempotent $(3, k, m)$-Latin trade for $2^{2 p-2} \leqslant m \leqslant 2^{2 p}$ and $14 \cdot 2^{p} \leqslant k \leqslant m-1$, showing the result.

Theorem 43. For $m \geqslant 5$, there exists an idempotent $(3, k, m)$-Latin trade for $5 \leqslant k \leqslant$ $m-1$ except possibly for those values in Table 1 and when $(k, m) \in\{(5,6),(5,10),(5,13)\}$.

Proof. Define $P(r)$ to be the statement "There exists idempotent $(3, k, m)$-Latin trades for each $5 \leqslant k \leqslant m-1$ and $2^{r-1}-6 \leqslant m \leqslant 2^{r}$ ".

Lemma 41 shows $P(r)$ is true for $9 \leqslant r \leqslant 18$. Assume for the sake of strong induction that $P(r)$ is true for $9 \leqslant r<R$, with $R \geqslant 19$. Then $P\left(\left\lceil\frac{R}{2}\right\rceil-1\right)$ and $P\left(\left\lceil\frac{R}{2}\right\rceil\right)$ are true, as $9 \leqslant\left\lceil\frac{R}{2}\right\rceil-1<R$. This makes the premise of Lemma 42 with $p=\left\lceil\frac{R}{2}\right\rceil$ true, and so there exists an idempotent $(3, k, m)$-Latin trade for $14 \cdot 2^{\left\lceil\frac{R}{2}\right\rceil} \leqslant k \leqslant m-1$ and $2^{R-1} \leqslant m \leqslant 2^{R}$, as $2^{2\left\lceil\frac{R}{2}\right\rceil-2} \leqslant 2^{R-1}$ and $2^{R} \leqslant 2^{2\left\lceil\frac{R}{2}\right\rceil}$. As $P(R-1)$ is true, we can apply Theorem 15 with $k^{\prime}=2^{R-2}$ and for each $k \in\left\{5, \ldots, 2^{R-2}-1\right\}$, which yields idempotent $(3, k, m)$-Latin trade for $5 \leqslant k \leqslant 2^{R-2}-1$ and $2^{R-1} \leqslant m \leqslant 2^{R}$. As $14 \cdot 2^{\left\lceil\frac{R}{2}\right\rceil} \leqslant 2^{R-2}-1$, this shows there exists an idempotent $(3, k, m)$-Latin trade for $5 \leqslant k \leqslant m-1$ and $2^{R-1} \leqslant m \leqslant 2^{R}$, and so $P(R)$ is true. By strong induction, $P(r)$ is true for $r \geqslant 9$. Theorem 22 and Lemma 41 complete the result when $5 \leqslant m<2^{8}-6$.

\section{Results}

As $\mathcal{I} \mathcal{S}_{m}^{\mu} \subseteq \mathcal{S}_{m}^{\mu}$, the results of Theorem 43 also yield the existence of $(3, k, m)$-Latin trades for identical values of $k$ and $m$. There are a few more non-idempotent $(3, k, m)$-Latin trades that we can find.

There does not exist a large set of idempotent Latin squares of order $n=6$, however there does exist a $(4,5,6)$-Latin trade given by:

\begin{tabular}{|c|c|c|c|c|c|}
\hline$(2,3,4,5)$ & $\bullet$ & $(1,4,5,3)$ & $(5,2,1,4)$ & $(3,5,2,1)$ & $(4,1,3,2)$ \\
\hline$\bullet$ & $(3,2,5,4)$ & $(6,3,4,5)$ & $(4,5,2,6)$ & $(5,6,3,2)$ & $(2,4,6,3)$ \\
\hline$(1,4,5,3)$ & $(4,5,3,6)$ & $(5,1,6,4)$ & $\bullet$ & $(6,3,1,5)$ & $(3,6,4,1)$ \\
\hline$(4,5,2,1)$ & $(5,6,4,2)$ & $\bullet$ & $(1,4,6,5)$ & $(2,1,5,6)$ & $(6,2,1,4)$ \\
\hline$(5,1,3,2)$ & $(6,3,2,5)$ & $(3,5,1,6)$ & $(2,6,5,1)$ & $(1,2,6,3)$ & $\bullet$ \\
\hline$(3,2,1,4)$ & $(2,4,6,3)$ & $(4,6,3,1)$ & $(6,1,4,2)$ & $\bullet$ & $(1,3,2,6)$ \\
\hline
\end{tabular}

Here, the partial Latin squares have been concatenated, so that cell $(r, c)$ has been filled with the ordered 4 -tuple given by $\left(t_{1}(r, c), t_{2}(r, c), t_{3}(r, c), t_{4}(r, c)\right)$, where the four partial Latin squares $T_{i}=\left[t_{i}(r, c)\right], 1 \leqslant i \leqslant 4$, form the $(4,5,6)$-Latin trade. Then this yields a non-idempotent $(3,5,6)$-Latin trade. 
Applying Theorem 7 of [2] to the combination of a $(3,5,6)$-Latin trade and a $(3,5,7)$ Latin trade yields a $(3,5,13)$-Latin trade. There exists a $(3, m, m)$-Latin trade for $m \geqslant 3$ by Theorem 11, and a $(3, m, 2 m)$-Latin trade by applying Theorem 7 of [2] to the combination of two $(3, m, m)$-Latin trades. Then the primary result of this paper, combined with previous results [2], can be written as the following theorem:

Theorem 44. For $m \geqslant 4$ there exists a $(3, k, m)$-Latin trade for $4 \leqslant k \leqslant m$ except, perhaps, for those unstared values in Table 1 and for $(k, m)=(4,11)$, and except for those values with $(k, m) \in\{(4,6),(4,7)\}$. For $m \geqslant 3$, there exists a $(3,3, m)$-Latin trade only when $3 \mid m$.

This leaves us with 194 exceptions for which we do not know if a $(3, k, m)$-Latin trade exists, and 2 exceptions when we know that there does not exist a $(3, k, m)$-Latin trade.

\section{$7 \quad$ Future work}

Given the relative success of finding base rows from Theorem 22, where the program terminated rather early within the search space, it seems reasonable that $(3, k, m)$-Latin trades with values in Table 1 could exist, and we can use this as evidence towards a conjecture:

Conjecture 45. There exists a $(3, k, m)$-Latin trade exactly when $k=3$ and $3 \mid m$, and when $4 \leqslant k \leqslant m$, except in the cases that $(k, m) \in\{(4,6),(4,7),(4,11)\}$.

It also seems that similar techniques used in this paper could be used to fill in the spectrum of $(4, k, m)$-Latin trades. In addition, it may be of interest to investigate the spectrum of circulant $(\mu, k, m)$-Latin trades.

A $\mu$-way Latin trade $\left(Q_{1}, \ldots, Q_{\mu}\right)$ can be said to be primary if there is no $\mu$-way Latin trade $\left(R_{1}, \ldots, R_{\mu}\right)$ such that $R_{\alpha} \subsetneq Q_{\alpha}$. A $\mu$-way Latin trade is said to be minimal if there is no partial Latin square $R \subsetneq Q_{1}$ such that there exists a 2-way trade $\left(R, R^{\prime}\right)$. Primary $(2, k, m)$-Latin trades were conjectured to exist for $3 \leqslant k \leqslant m$ in [8]. It would be of interest to investigate primary and minimal $(\mu, k, m)$-Latin trades in the future.

\section{Acknowledgements}

The first author would like to thank the hospitality of Soochow University where partial research was done during his visit. The authors would also like to thank Prof. L. Zhu for many suggestions, and D. Donovan for extensive proofreading. 


\begin{tabular}{|c|c|}
\hline$m$ & $k$ \\
\hline 22 & 19 \\
\hline 23 & $19,20,21$ \\
\hline 26 & $19,21,23$ \\
\hline 29 & $19,20,21,22,23,24,25,26,27$ \\
\hline 31 & $19,20,21,22,23,24,25,26,27,28,29$ \\
\hline 34 & $19,21,23,25,27,29,31$ \\
\hline 37 & $33,34,35$ \\
\hline 38 & $19^{*}, 21,23,25,27,29,31,33,35$ \\
\hline 41 & $35,36,37,38,39$ \\
\hline 43 & $36,37,38,39,40,41$ \\
\hline 46 & $27,29,31,33,35,37,39,41,43$ \\
\hline 53 & 50,51 \\
\hline 58 & $29^{*}, 31,33,35,37,39,41,43,45,47,49,51,53,55$ \\
\hline 59 & $55,56,57$ \\
\hline 62 & $31^{*}, 33,35,37,39,41,43,45,47,49,51,53,55,57,59$ \\
\hline 74 & $35,37^{*}, 39,41,43,45,47,49,51,53,55,57,59,61,63,65,67,69,71$ \\
\hline 82 & $41^{*}, 43,45,47,49,51,53,55,57,59,61,63,65$ \\
\hline 86 & $51,53,55,57,59,61,63,65,67,69$ \\
\hline 94 & $51,53,55,57,59,61,63,65$ \\
\hline 106 & $53^{*}, 55,57,59,61,63,65,67,69$ \\
\hline 122 & $59,61,63,65,67,69,71,73,75,77$ \\
\hline 134 & $67^{*}, 69,71,73,75,77,79,81,83,85,87,89,91,93$ \\
\hline 146 & $83,85,87,89,91,93,95,97,99,101$ \\
\hline 158 & $83,85,87,89,91$ \\
\hline 194 & $97^{*}, 99,101$ \\
\hline
\end{tabular}

Table 1: Values where no idempotent $(3, k, m)$-Latin trade is known to exist with $6 \leqslant$ $k \leqslant m-1$. The starred values indicate when a non-idempotent $(3, k, m)$-Latin trade is known to exist. 


\section{References}

[1] P. Adams, E.J. Billington, D.E. Bryant, and E.S. Mahmoodian. The three-way intersection problem for Latin squares. Discrete Math., 243(1-3):1-19, 2002.

[2] B. Bagheri Gh., D. Donovan, and E.S. Mahmoodian. On the existence of 3-way k-homogeneous Latin trades. Discrete Math., 312(24):3473-3481, 2012.

[3] B. Bagheri Gh. and E.S. Mahmoodian. On the existence of $k$-homogeneous Latin bitrades. Util. Math., 85:333-345, 2011.

[4] R. Bean, H. Bidkhori, M. Khosravi, and E.S. Mahmoodian. k-homogeneous Latin trades. Bayreuth. Math. Schr., 74:7-18, 2005.

[5] N.J. Cavenagh. A uniqueness result for 3-homogeneous Latin trades. Comment. Math. Univ. Carolin., 47:337-358, 2006.

[6] N.J. Cavenagh, D. Donovan, and A. Drápal. 3-homogeneous Latin trades. Discrete Math., 300(1-3):57-70, 2005.

[7] N.J. Cavenagh, D. Donovan, and A. Drápal. 4-homogeneous Latin trades. Australas. J. Combin., 32:285-303, 2005.

[8] N.J. Cavenagh and I.M. Wanless. On the number of transversals in Cayley tables of cyclic groups. Discrete Appl. Math., 158(2):136-146, 2010.

[9] Y. Chang. The existence of a large set of idempotent quasigroups of order $62 . J$. Combin. Math. Combin. Comput., 32:213-218, 2000.

[10] Y. Chang. The spectrum for large sets of idempotent quasigroups. J. Combin. Des., $8(2): 79-82,2000$.

[11] C.J. Colbourn and J.H. Dinitz., editors. Handbook of Combinatorial Designs. Discrete Mathematics and Its Applications (Boca Raton). Chapman \& Hall/CRC, Boca Ratan, FL, second edition, 2007.

[12] M.J. Grannell, T.S. Griggs, and M. Knor. Biembeddings of symmetric configurations and 3-homogeneous Latin trades. Comment. Math. Univ. Carolin., 49(3):411-420, 2008.

[13] C. Hämäläinen. Partitioning 3-homogeneous Latin bitrades. Geom. Dedicata, 133:181-193, 2008.

[14] J.G. Lefevre, D. Donovan, and A. Drápal. Permutation representation of 3 and 4-homogeneous Latin bitrades. Fund. Inform., 84(1):99-110, 2008.

[15] T.G. Marbach, 3-way $k$-homogeneous Latin trades - programs a and b. https://github.com/triliu/3WaykHomogeneousLatinTrades

[16] T.G. Marbach. On the intersection of three or four transversals of the back circulant Latin square $B_{n}$. arXiv:1407.5174v2, 2015.

[17] L. Teirlinck and C.C. Lindner. The construction of large sets of idempotent quasigroups. European J. Combin., 9(1):83-89, 1988.

[18] W.D. Wallis. Combinatorial Designs. Monogr. Textbooks Pure Appl. Math., 118, 1988. 